\title{
Aspectos relevantes da visita pré-operatória de Enfermagem: benefícios para o paciente e para a assistência
}

\author{
Relevant aspects of the preoperative Nursing visit: benefits for the patient and care
}

Aspectos relevantes de la visita preoperatoria de Enfermería: beneficios para el paciente y para la asistencia

Ilana Maria Brasil do Espírito Santo ${ }^{1}$, Francisco Lucas de Lima Fontes ${ }^{2 *}$, Patrícia Maria do Espírito Santo $^{3}$, Anakelle Oliveira dos Santos ${ }^{4}$, Elisangela Póvoas de Oliveira ${ }^{1}$, Sandra Alves Mendes Noleto Velozo $^{5}$, Brenda Lícia Martins da Silva ${ }^{2}$, Ilana Isla Oliveira ${ }^{2}$, Bruna Lira Santos ${ }^{2}$, Wesley Brandolee Bezerra Fernandes ${ }^{2}$, Jadson Farias de Sousa ${ }^{2}$, Lucilene da Silva Silva ${ }^{1}$, Rafael da Silva Nascimento², Adalberto Moreira da Silva Júnior², Luana Letícia Silva Carvalho.

\section{RESUMO}

Objetivo: Identificar os aspectos relevantes da visita pré-operatória de Enfermagem de modo a evidenciar seus benefícios para o paciente e para a assistência. Métodos: Trata-se de uma revisão bibliográfica narrativa descritiva, realizada por meio das bases de dados Biblioteca Virtual em Saúde (BVS) e Base de Dados de Enfermagem (BDEnf) e do buscador acadêmico Google Acadêmico, utilizando os descritores "cirurgia", "enfermagem" e "visita pré-operatória". Foram selecionados oito artigos para discussão, além de um Manual de Práticas Recomendadas. Resultados: Educação para a saúde, preparo físico e emocional, prevenção de complicações no pós-operatório e diversas competências presentes no pré-operatório mediato e imediato foram alguns dos aspectos relevantes identificados. Considerações Finais: A realização sistemática da visita pré-operatória fornece subsídios ao enfermeiro para que haja o correto e integral preparo do paciente ao procedimento anestésico-cirúrgico, qualificando-o para vivenciar o pré-operatório mais tranquilo possível e um pós-operatório isento de complicações e danos, tornando esse processo estressante menos traumático.

Palavras-chave: Enfermagem, Cirurgia, cuidados pré-operatórios.

\begin{abstract}
Objective: To identify the aspects of the preoperative nursing consultation to show its benefits for the patient and the care. Methods: This is a descriptive narrative bibliographical review, based on the databases of the Virtual Health Library (VHL) and the Nursing Database (BDEnf) and the academic search engine Google Academic, using the descriptors "surgery" , "Infirmary and preoperative visit. There were also articles for discussion, in addition to a Handbook of Best Practices. Results: Education for health, physical and emotional preparation, prevention of complications, non-postoperative and in various ways, no preoperative information. Final considerations: The systematic function of preoperative care provides the nurses with the possibility of performing the integral preparation of the patient to the anesthetic-surgical procedure, qualifying him to experience the most pre-operative possible and a post-operative ware and damages, using the stressful process less traumatic.
\end{abstract}

Keywords: Nursing, Surgery, Preoperative care.

${ }^{1}$ Centro Universitário UNINOVAFAPI. Teresina, Piauí, Brasil.

2 Faculdade UNINASSAU - Campus Redenção. Teresina, Piauí, Brasil. *E-mail:lucasfontesenf@hotmail.com

3 Universidade Estadual do Piauí. Teresina, Piauí, Brasil.

${ }^{4}$ Centro Universitário Santo Agostinho. Teresina, Piauí, Brasil.

${ }^{5}$ NOVAUNESC Faculdade. Teresina, Piauí, Brasil.

SUBMETIDO EM: $3 / 2019$ 


\section{RESUMEN}

Objetivo: Identificar los aspectos relevantes de la visita preoperatoria de Enfermería de modo a evidenciar sus beneficios para el paciente y para la asistencia. Métodos: Se trata de una revisión bibliográfica narrativa descriptiva, realizada por medio de las bases de datos Biblioteca Virtual en Salud (BVS) y Base de Datos de Enfermería (BDEnf) y del buscador académico Google Académico, utilizando los descriptores "cirugía", "enfermería" y "visita preoperatoria". Se seleccionaron ocho artículos para discusión, además de un manual de prácticas recomendadas. Resultados: Educación para la salud, preparación física y emocional, prevención de complicaciones en el postoperatorio y diversas competencias presentes en el preoperatorio mediato e inmediato fueron algunos de los aspectos relevantes identificados. Consideraciones Finales: La realización sistemática de la visita preoperatoria proporciona subsidios al enfermero para que haya la correcta e integral preparación del paciente al procedimiento anestésico-quirúrgico, calificándolo para vivir el preoperatorio más tranquilo posible y un postoperatorio exento de complicaciones y daños, haciendo que este proceso estresante menos traumático.

Palabras-clave: Enfermería, Cirugía, Cuidados preoperatorios.

\section{INTRODUÇÃO}

A existência de qualquer processo patológico desperta no indivíduo uma série de preocupações que o dirige a sentimentos de medo e impotência. A necessidade de submissão a um procedimento cirúrgico torna intenso esses sentimentos e coloca o indivíduo diante de uma série de questionamentos acerca do procedimento ao qual terá que submeter-se, suas possíveis complicações e sua recuperação. Além disso, as dúvidas levantadas pelo paciente leva-o a caracterizar o ato cirúrgico como uma situação crítica que implicará numa indefinição de futuros fatos (BARBOSA JA, 2014).

Todo o perioperatório, período que compreende o pré-operatório, transoperatório, intraoperatório, recuperação pós-anestésica e pós-operatório, mostra-se relevante nos cuidados despendidos ao paciente cirúrgico, contudo é na fase pré-operatória que o sujeito a ser cirurgia do encontra-se mais vulnerável em suas necessidades fisiológicas e psicológicas, estando predisposto a um desequilíbrio emocional (OLIVEIRA MM e MEDONÇA KM, 2014).

A assistência que a Enfermagem presta ao paciente antes do procedimento cirúrgico é importante, pois prepara o físico e, sobretudo o emocional do cliente. O enfermeiro, diante desse contexto, deve estar apto a praticar as duas vertentes principais da visita pré-operatória: as orientações ao paciente, voltadas à educação e ao preparo emocional, e os preparativos físicos para a cirurgia (SILVA DO e TELES NB, 2013).

Para que ocorra a continuidade dos cuidados prestados, a Enfermagem dispõe da Sistematização da Assistência de Enfermagem Perioperatória (SAEP), um recurso da profissão aplicado ao paciente cirúrgico, que possibilita a operacionalização da assistência prestada em todo o período perioperatório (BARBOSA JA, 2014). A profissão detém respaldo legal para aplicação da SAEP, amparada na Resolução № 358 de 2009 do Conselho Federal de Enfermagem, que garante a implementação da Sistematização da Assistência de Enfermagem (SAE) em instituições públicas e privadas em que ocorram o cuidado de Enfermagem (CONSELHO FEDERAL DE ENFERMAGEM, 2009).

Dentre as finalidades da SAEP estão o apoio ao paciente e à família no entendimento e preparo ao ato anestésico-cirúrgico, redução dos riscos derivados do espaço físico do setor e do uso de materiais e equipamentos úteis à execução da cirurgia e oferecimento de uma assistência segura, por tratar-se de uma metodologia (FERRAZ KMC et al., 2016).

A SAEP fundamenta-se em cinco etapas inter-relacionadas, a saber: visita pré-operatória de Enfermagem com histórico e exame físico, planejamento da assistência pré-operatória, implementação das intervenções, visita pós-operatória e reformulação da assistência, esta última para os casos em que os resultados obtidos diferirem daqueles esperados (MALAGUTTI W, BONFIM IM, 2011). A execução da SAEP é uma atividade 
exclusiva do enfermeiro e requer desse profissional ampla compreensão sobre o holismo perioperatório (SOBECC, 2013).

A fim de guiar, embasar e dar suporte adequado ao enfermeiro que implementa a SAEP e consequentemente realiza a visita pré-operatória, buscou-se neste estudo sintetizar o conhecimento já produzido sobre a temática. Acredita-se que estudos sobre a assistência do enfermeiro na visita préoperatória de Enfermagem, essencial à SAEP, norteiam a prática clínica. Diante do explanado, o objetivo do presente estudo foi identificar os aspectos relevantes da visita pré-operatória de Enfermagem de modo a evidenciar seus benefícios para o paciente e para a assistência.

\section{MÉTODOS}

Trata-se de uma revisão de literatura do tipo bibliográfica narrativa descritiva. A escolha do tema é de fundamental importância na produção de toda pesquisa científica. Na definição do tema do presente estudo foi considerado primordialmente o interesse dos autores pela área de Enfermagem Cirúrgica.

Foi realizada uma busca bibliográfica nas bases de dados Biblioteca Virtual em Saúde (BVS), Banco de Dados de Enfermagem (BDEnf) e no buscador acadêmico Google Acadêmico. As palavras-chave do Descritores em Ciências da Saúde (DeCS) adotados para a busca dos referenciais foram: "cirurgia", "enfermagem" e "visita pré-operatória". Os critérios para inclusão dos artigos para discussão foram: artigos disponíveis na íntegra, em língua portuguesa, publicados de 2013 a 2018. Os critérios de exclusão foram artigos incompletos, monografias, dissertações e teses. Foram incluídos, após aplicação dos critérios de inclusão oito artigos para discussão. Além dos artigos, também se considerou relevante para discussão o Manual de Práticas Recomendadas da Associação Brasileira de Enfermeiros de Centro Cirúrgico, Recuperação Anestésica e Centro de Material e Esterilização (SOBECC).

A coleta de dados obedeceu às seguintes etapas: leitura meticulosa de todos artigos selecionados (leitura flutuante com vistas a verificação de relevância do artigo para o estudo em questão); leitura seletiva (apreciação mais aprofundada das partes essenciais à construção da discussão do estudo); apontamento dos dados extraídos das fontes em material específico. A análise dos materiais teve o objetivo de ordenar e simplificar as informações contidas nas fontes, de forma que estas possibilitem a obtenção das respostas ao problema desta pesquisa.

\section{RESULTADOS E DISCUSSÃO}

A fase pré-operatória é dividida em mediata e imediata. A etapa mediata consiste no preparo semanas ou meses antes do procedimento, como regime alimentar específico, diminuição do tabagismo ou controle da diabetes. Já a fase imediata inicia-se 24 horas antes do procedimento cirúrgico, quando normalmente são realizados os preparos específicos para a cirurgia, como o jejum oral absoluto, tricotomia da região a ser cirurgia (realizada no máximo duas horas antes do procedimento), higiene corporal, remoção de adornos metálicos, próteses dentárias, lentes de contato, higiene oral e esvaziamento vesical e intestinal antes de encaminhamento para o procedimento cirúrgico (SOBECC, 2013).

O enfermeiro, junto da sua equipe, deve trabalhar recursos facilitadores para que as atividades exercidas no centro cirúrgico sejam adaptadas à singularidade de cada paciente, com a finalidade de uma assistência efetiva. Diante das inúmeras atribuições do enfermeiro perioperatório, destaca-se a visita realizada antes do procedimento cirúrgico, indispensável na avaliação, detecção e solução de problemas identificados, além de muitas vezes ser a promotora do primeiro contato enfermeiro-paciente (ROCHA DR e IVO OP, 2015).

A visita pré-operatória de Enfermagem é o início de execução da SAEP. Esta primeira fase é alcançada por meio de visita ao paciente, análise do prontuário e comunicação com o enfermeiro do posto de internação. É uma atividade que objetiva compreender a realidade do cliente e preservar a relação enfermeiro-paciente, além de nortear, investigar e encaminhar os problemas detectados a outros profissionais, quando necessário (ASCARI RA, 2014). 
Conforme seleção dos artigos utilizados, segue a Quadro $1 \mathrm{com}$ distribuição de títulos dos estudos, autores/ano, tipos de estudo e principais conclusões.

Quadro 1 - Resultados dos artigos selecionados de acordo com título, autores/ano, tipo/abordagem de estudo e conclusões.

\begin{tabular}{|c|c|c|c|}
\hline TÍTULO & AUTORES/ANO & $\begin{array}{l}\text { TIPO DE } \\
\text { ESTUDO }\end{array}$ & CONCLUSÃO \\
\hline $\begin{array}{l}\text { Percepções do paciente } \\
\text { cirúrgico no período pré- } \\
\text { operatório acerca da } \\
\text { assistência }\end{array}$ & $\begin{array}{l}\text { ASCARI RA, } \\
\text { NEISS M, } \\
\text { SARTORI AA et } \\
\text { al (2013) }\end{array}$ & $\begin{array}{c}\text { Estudo } \\
\text { qualitativo }\end{array}$ & $\begin{array}{l}\text { A compreensão da percepção do paciente } \\
\text { cirúrgico no período pré-operatório pode agregar } \\
\text { subsídios para destacar que uma das formas de } \\
\text { prestar assistência é por meio da sistematização } \\
\text { da assistência de enfermagem, aliada a uma rotina } \\
\text { de orientações, realizada pelo enfermeiro da } \\
\text { clínica cirúrgica. }\end{array}$ \\
\hline $\begin{array}{l}\text { Prática do enfermeiro no } \\
\text { cuidado ao paciente no } \\
\text { pré-operatório imediato de } \\
\text { cirurgia eletiva }\end{array}$ & $\begin{array}{l}\text { SENA AC, } \\
\text { NASCIMENTO } \\
\text { ERP, MAIA ARCR } \\
(2013) \\
\end{array}$ & $\begin{array}{c}\text { Estudo } \\
\text { qualitativo }\end{array}$ & $\begin{array}{l}\text { Concluiu-se que os cuidados foram direcionados } \\
\text { aos aspectos físicos em detrimento do psicológico, } \\
\text { em discordância com a metodologia assistencial } \\
\text { adotada no hospital, sustentada pela Teoria das } \\
\text { Necessidades Humanas Básicas. }\end{array}$ \\
\hline $\begin{array}{l}\text { Reflexão sobre o cuidado } \\
\text { dispensado ao paciente } \\
\text { cirúrgico no perioperatório }\end{array}$ & $\begin{array}{l}\text { ASCARI RA } \\
(2014)\end{array}$ & $\begin{array}{l}\text { Relato de } \\
\text { experiência }\end{array}$ & $\begin{array}{l}\text { Considerar a intervenção anestésico-cirúrgica e o } \\
\text { cuidado desse processo em situações singulares } \\
\text { para o paciente é uma questão a ser trabalhada } \\
\text { com toda a equipe assistencial. Contudo, são } \\
\text { necessárias discussões em que se busque } \\
\text { despertar, estimular e apoiar uma assistência } \\
\text { humanística e implementar mudança de postura } \\
\text { entre os profissionais com re(educação) das } \\
\text { práticas de cuidado e valorizaçãa do ser humano. }\end{array}$ \\
\hline $\begin{array}{l}\text { As orientações } \text { de } \\
\text { enfermagem e sua } \\
\text { influência nos níveis de } \\
\text { ansiedade dos pacientes }\end{array}$ & $\begin{array}{l}\text { COSTA TMN e } \\
\text { SAMPAIO CEP } \\
\text { (2015) }\end{array}$ & $\begin{array}{c}\text { Estudo } \\
\text { quantitativo }\end{array}$ & $\begin{array}{l}\text { Conclui-se que as orientações de enfermagem no } \\
\text { pré-operatório exercem importância fundamental } \\
\text { tanto para o cliente como para toda a equipe que } \\
\text { participa desse momento, com vistas ao bem-estar }\end{array}$ \\
\hline
\end{tabular}
cirúrgicos hospitalares

Assistência de enfermagem no préoperatório e sua influência no pós-operatório: uma percepção do cliente

$\begin{array}{cl}\text { ROCHA DR e IVO } & \text { Estudo } \\ \text { OP (2015) } & \text { quantitativo }\end{array}$
e à redução da ansiedade no momento cirúrgico. $\mathrm{O}$ estudo mostrou que, a carência de informações/orientações prestadas pelo enfermeiro na fase pré-operatória dificulta 0 entendimento e provocam equívocos, neste sentido há uma necessidade de implementação de novas práticas, como a prescrição de enfermagem, visando um atendimento qualificado e minimizando possíveis complicações.

Concluiu-se que questões como comodismo ou por pacientes no préoperatório
Sentimentos vivenciados

ROCHA NMC, SILVA FAA, ROCHA RC et al. (2016)

\begin{tabular}{lcc}
\hline $\begin{array}{l}\text { Construção coletiva de um } \\
\text { instrumento de cuidados } \\
\text { de Enfermagem a } \\
\text { pacientes no pré- } \\
\text { operatório imediato }\end{array}$ & $\begin{array}{r}\text { SENA AC, } \\
\text { NASCIMENTO MAIA ARCR } \\
\text { et al. (2017) }\end{array}$ & $\begin{array}{c}\text { Estudo } \\
\text { qualitativo }\end{array}$ \\
\hline $\begin{array}{l}\text { Avaliação do estado de } \\
\text { ansiedade pré-operatória }\end{array}$ & $\begin{array}{c}\text { MELCHIOR LMR, } \\
\text { BARRETO RASS, } \\
\text { em pacientes cirúrgicos } \\
\text { ALENCAR LKB et } \\
\text { hospitalizados }\end{array}$ & $\begin{array}{c}\text { Estudo } \\
\text { quantitativo }\end{array}$ \\
\hline
\end{tabular}

Estudo técnicas mecânicas contínuas podem ser revistas para que a enfermagem como um todo possa atuar de modo a atenuar todos os sentimentos negativos e exacerbados que o cliente possa vir a relatar ou expressar. A versão final do instrumento ficou constituída por nove cuidados agrupados em duas categorias: orientação/ensino no pré-operatório e cuidado cirúrgico no pré-operatório.
A maioria dos pacientes que aguardavam cirurgia se apresentaram ansiosos e tiveram sinais e sintomas que repercutiram no seu bem-estar e induziram a um sofrimento psicobiológico.

Fonte: próprios autores, 2019.

$\mathrm{Na}$ atenção ao paciente em pré-operatório, além do preparo deste, a equipe de Enfermagem também é responsável por avaliar aspectos importantes como a classificação da cirurgia quanto ao porte, tempo necessário ao procedimento, tipo de anestesia a ser utilizada, estado físico do cliente, complexidade e/ou 
estágio da doença cirúrgica, nutrição, riscos transoperatórios e possíveis complicações no pós-operatório. É de crucial importância pesar os prós e os contras dos riscos existentes de cada procedimento cirúrgico, de modo a evitar ou restringir prováveis complicações (SENA AC et al., 2013).

Para Sena AC et al. (2017), as atividades que o enfermeiro desenvolve devem contemplar os aspectos físicos, psicológicos, sociais, culturais ou espirituais da condição humana, a fim de melhorar a qualidade de vida dos pacientes. O cumprimento de um cuidado cirúrgico de Enfermagem seguro e com a devida qualidade se inicia na etapa pré-operatória, que compreende desde o momento em que o paciente opta pela cirurgia e vai até a administração da anestesia.

Além de todo o preparo físico necessário à submissão a um procedimento cirúrgico, por vezes o enfermeiro exerce função importante na educação emocional do paciente. A visita pré-operatória de Enfermagem favorece a percepção sobre o estado de apreensão do paciente frente ao momento cirúrgico e possibilita a implementação de intervenções que visem a diminuição da ansiedade (COSTA TMN e SAMPAIO CEP, 2015).

O estudo de Melchior LMR (2018) mostrou que grande parte dos pacientes que aguardavam cirurgia eletiva apresentaram sentimentos ansiosos, o que desencadeou sintomas que afetaram o bem-estar e provocaram sofrimento psicobiológico. No estudo, a prevalência de ansiedade pré-operatória entre os 200 pacientes estudados foi de $53 \%$.

O estudo de Ascari RA et al. (2013) revelou um grave problema: orientações pré-operatórias executadas exclusivamente por profissionais médicos. Neste estudo também ficou claro por meio dos discursos dos participantes raras ocasiões em que o enfermeiro exercia seu papel de educador no preparo físico do paciente a fim de evitar complicações pós-operatórias. Isso evidencia a importância de a profissão fazer-se presente nesta etapa primordial do período perioperatório, pois ao passo em que favorece o vínculo enfermeiropaciente também repercute na qualidade da assistência oferecida.

Segundo a Associação Brasileira de Enfermeiros de Centro Cirúrgico, Recuperação Anestésica e Centro de Material e Esterilização (2013), quando realizada pelo enfermeiro, a visita pré-operatória fornece elementos necessários ao seu julgamento clínico para implementação de ações da assistência individual prestada. Isso permite ao profissional a tomada de condutas importantes como a identificação dos diagnósticos de Enfermagem, o comprometimento com intervenções palpáveis à condição de saúde do paciente e a realização de avaliação do que foi implementado. Essas ações melhoram a qualidade do cuidado e a relação paciente-família-profissionais durante execução da SAEP, além de possibilitar momentos de orientação durante o contato.

Paciente bem orientado é paciente cooperativo e sabedor de suas condutas, e isso contribui no próprio tratamento e recuperação pós-cirúrgica pois desta forma o cliente sente-se protagonista de sua reabilitação exercendo o autocuidado. Assim, faz-se necessário que o enfermeiro compreenda o paciente como um ser singular, sujeito que demanda cuidados diferenciados dos demais e possui diferentes níveis de conhecimentos e sentimentos e que este profissional exerça seu papel de educador em saúde orientando os pacientes cirúrgicos (ROCHA NMC et al., 2016).

\section{CONSIDERAÇÕES FINAIS}

A realização sistemática da primeira etapa da SAEP fornece subsídios ao enfermeiro para que haja o correto e integral preparo do paciente ao procedimento anestésico-cirúrgico, qualificando-o para vivenciar o pré-operatório mais tranquilo possível e um pós-operatório isento de complicações e danos, tornando esse processo estressante menos traumático. Educação para a saúde, preparo físico e emocional, prevenção de complicações no pós-operatório e diversas competências presentes no pré-operatório mediato e imediato foram alguns dos aspectos relevantes identificados. Observou-se por meio da busca dos artigos um número reduzido de publicações sobre essa temática, o que demonstra a necessidade de maiores estudos, a fim de que se construa uma assistência baseada em comprovações científicas, motivando de maneira consistente $o$ cuidar na área da Enfermagem Cirúrgica. 


\section{REFERÊNCIAS}

1. ASCARI RA. Reflexão sobre o cuidado dispensado ao paciente cirúrgico no perioperatório. Revista Uningá, 2014; 19(2): 33-36.

2. ASCARI RA et al. Percepções do paciente cirúrgico no período pré-operatório acerca da assistência de enfermagem. Revista de Enfermagem da UFPE, 2013; 7(4): 1136-1144.

3. ASSOCIAÇÃO BRASILEIRA DE ENFERMEIROS DE CENTRO CIRÚRGICO, RECUPERAÇÃO ANESTÉSICA E CENTRO DE MATERIAL E ESTERILIZAÇÃO (SOBECC). Práticas recomendadas SOBECC. $6^{a}$ ed. São Paulo: Manole, 2013.

4. CONSELHO FEDERAL DE ENFERMAGEM (COFEN). Resolução no 358, de 15 de outubro de 2009. COFEN, 2009.

5. COSTA TMN, SAMPAIO CEP. As orientações de enfermagem e sua influência nos níveis de ansiedade dos pacientes cirúrgicos hospitalares. Revista de Enfermagem da UERJ, 2015; 23(2): 260-265.

6. BARBOSA JA. Visita pré-operatória de Enfermagem: Contribuições no pré e pós-operatório. Trabalho de Conclusão de Curso (Graduação em Enfermagem) - Universidade Estadual da Paraíba, Campina Grande, 2014.

7. FERRAZ KMC et al. Percepção dos graduandos de enfermagem sobre a sistematização da assistência de enfermagem perioperatória. Revista de Enfermagem da UFPE, 2016; 10(6): 2108-2115.

8. MALAGUTTI W, BONFIM IM. Enfermagem em centro cirúrgico: atualidades e perspectivas no ambiente cirúrgico. $2^{a}$ ed. São Paulo: Martinari, 2011.

9. MELCHIOR LMR et al. Avaliação do estado de ansiedade pré-operatória em pacientes cirúrgicos hospitalizados. Revista de Enfermagem da UFJF, 2018; 4(2): 107-114.

10. OLIVEIRA MM, MENDONÇA KM. Análise da visita pré-operatória de enfermagem: revisão integrativa. Revista SOBECC, 2014; 19(3): 164-172.

11. ROCHA DR, IVO OP. Assistência de enfermagem no pré-operatório e sua influência no pós-operatório: uma percepção do cliente. Revista Enfermagem Contemporânea, 2015; 4(2): 170-178.

12. ROCHA NMC et al. Sentimentos vivenciados por pacientes no pré-operatório. Revista Interdisciplinar, 2016; 9(2): 178-186.

13. SENA AC et al. Construção coletiva de um instrumento de cuidados de Enfermagem a pacientes no préoperatório imediato. Revista Baiana de Enfermagem, 2017; 31(1): e20506.

14. SENA AC et al. Prática do enfermeiro no cuidado ao paciente no pré-operatório imediato de cirurgia eletiva. Revista Gaúcha de Enfermagem, 2013; 34(3): 132-137.

15. SILVA DO, TELES NB. A relevância da visita pré-operatória no processo da cirurgia cardíaca e suas implicações para a enfermagem: uma revisão integrativa de literatura. Revista Diálogos Acadêmicos, 2013; 2(2): 109-115. 